\title{
On-line scheduling to minimize average completion time revisited
}

\author{
Nicole Megow ${ }^{\mathrm{a}, *, 1}$, Andreas S. Schulz ${ }^{\mathrm{b}}$ \\ ${ }^{a}$ Institut für Mathematik, Technische Universität Berlin, Sekr. MA 6-1, Strasse des 17. Juni 136, Berlin 10623, Germany \\ ${ }^{\mathrm{b}}$ Sloan School of Management, Massachusetts Institute of Technology, Office E53-361, 77 Massachusetts Avenue, \\ Cambridge, MA 02139-4307, USA
}

Received 4 October 2003; received in revised form 3 November 2003; accepted 18 November 2003

\begin{abstract}
We consider the scheduling problem of minimizing the average-weighted completion time on identical parallel machines when jobs are arriving over time. For both the preemptive and the nonpreemptive setting, we show that straightforward extensions of Smith's ratio rule yield smaller competitive ratios than the previously best-known deterministic on-line algorithms. (c) 2003 Elsevier B.V. All rights reserved.
\end{abstract}

Keywords: Scheduling; On-line algorithm; Approximation algorithm; Competitive analysis

\section{Introduction}

Model. We consider the problem of scheduling jobs arriving over time on-line on identical parallel machines to minimize the sum of weighted completion times. Each of the $m$ machines can process only one of the $n$ jobs at a time. Each job $j$ of a given instance has a positive processing time $p_{j}>0$ and a nonnegative weight $w_{j} \geqslant 0$. We learn about these job data only at the job's release date $r_{j} \geqslant 0$, which is not known in advance, either. If $C_{j}$ denotes the completion time of job $j$ in a

\footnotetext{
* Corresponding author.

E-mail addresses: nmegow@math.tu-berlin.de (N. Megow), schulz@mit.edu (A.S. Schulz).

${ }^{1}$ Supported by the DFG Research Center "Mathematics for key technologies" (FZT 86) in Berlin, Germany. Part of this research was performed while this author was visiting the Operations Research Center at the Massachusetts Institute of Technology.
}

feasible schedule, the corresponding objective function value is $\sum_{j=1}^{n} w_{j} C_{j}$. We consider both the preemptive and the nonpreemptive machine environments. In the preemptive setting, the processing of a job may be suspended and resumed later on any machine at no extra cost. In contrast, once a job is started in the nonpreemptive mode, it must be processed on the same machine without any interruption until its completion. In scheduling notation [6], the corresponding off-line problems are denoted by $\mathrm{P} \mid r_{j}$, pmtn $\mid \sum w_{j} C_{j}$ and $\mathrm{P}\left|r_{j}\right| \sum w_{j} C_{j}$, respectively. Already the analogous single-machine problems are NP-hard $[9,10]$.

However, instances of $1 \| \sum w_{j} C_{j}$ are optimally solved by Smith's weighted shortest processing time (WSPT) rule, which sequences jobs in nonincreasing order of their weight-to-processing-time ratios [17]. For convenience, we assume that the jobs are indexed in this order so that $w_{1} / p_{1} \geqslant w_{2} / p_{2} \geqslant \cdots \geqslant w_{n} / p_{n}$. Moreover, we say that a job with a smaller 
index has higher priority than one with a larger index.

The quality of on-line algorithms is typically assessed by their worst-case performance, expressed as the competitive ratio [16]. A $\rho$-competitive algorithm provides for any instance a solution with an objective function value of at most $\rho$ times the value of an optimal off-line solution.

Main results. We show in Section 2 that a natural extension of the WSPT rule to preemptive scheduling on identical parallel machines with release dates is 2-competitive, and this bound is tight. The idea is to interrupt currently active jobs of lower priority whenever new high-priority jobs arrive and not enough machines are available to accommodate the arrivals.

When preemption is not allowed, a straightforward extension of this scheme is to start the currently available job of highest priority whenever a machine becomes idle. However, this rule does not directly lead to a bounded competitive ratio. In fact, consider a single-machine instance in which a job of high priority is released right after the start of a long lower-priority job. Therefore, we first modify the release date of each job such that it is equal to a certain fraction of its processing time, if necessary. If we now start a long job $j$ and a high-priority job becomes available shortly thereafter, the ill-timed choice of starting $j$ can be accounted for by the fact that the high-priority job has a release date or processing time at least as large as a fraction of $p_{j}$. Therefore, its delay is bounded by its own contribution to the objective function in any feasible schedule. We consider a family of alike algorithms in Section 3 and show that the best one is 3.28-competitive. In this case, we cannot show that our analysis is tight, but the remaining gap is at most 0.5 .

Related work. Lu et al. [11] introduced a related class of 2-competitive algorithms, which use similar waiting strategies for the on-line variant of the single-machine problem $1\left|r_{j}\right| \sum C_{j}$. In fact, the idea of boosting release dates was used before by Hoogeveen and Vestjens [8] and Stougie (cited in [18]), who delayed the release date of each job $j$ until time $\max \left\{r_{j}, p_{j}\right\}$ and $r_{j}+p_{j}$, respectively. Anderson and Potts [2] extended both, Hoogeveen and Vestjens' algorithm and its competitive ratio of 2 , to the setting of arbitrary nonnegative job weights. These results are best possible since Hoogeveen and Vestjens also proved that no nonpreemptive deterministic on-line algorithm can achieve a competitive ratio better than 2.

Phillips et al. [12] presented another on-line algorithm for $1\left|r_{j}\right| \sum C_{j}$, which converts a preemptive schedule into a nonpreemptive one of objective function value at most twice that of the preemptive schedule. Since Schrage's shortest remaining processing time (SRPT) rule [13] works on-line and produces an optimal preemptive schedule for the single-machine problem, it follows that Phillips, Stein and Wein's algorithm has competitive ratio 2 as well. The conversion factor is $3-1 / m$ if applied to identical parallel machines, but the corresponding preemptive problem is NP-hard in this case. However, Chekuri et al. [3] noted that by sequencing jobs nonpreemptively in the order of their completion times in the optimal preemptive schedule on a single machine of speed $m$ times as fast as that of any one of the $m$ parallel machines, one obtains a $(3-1 / m)$-competitive algorithm for the on-line version of $\mathrm{P}\left|r_{j}\right| \sum C_{j}$. For the same problem, Lu et al. [11] gave a $2 \alpha$-competitive algorithm, where $\alpha$ is the competitive ratio of the direct extension of the SRPT rule to identical parallel machines. Phillips et al. [12] showed that this rule is 2-competitive, but a smaller value of $\alpha$ has not been ruled out.

In any case, the hitherto best known deterministic on-line result for the corresponding scheduling problems with arbitrary job weights, $P\left|r_{j}, \operatorname{pmtn}\right| \sum w_{j} C_{j}$ and $P\left|r_{j}\right| \sum w_{j} C_{j}$ was a $(4+\varepsilon)$-competitive algorithm by Hall et al. [7], which was given as part of a more general on-line framework. For $1\left|r_{j}, \operatorname{pmtn}\right| \sum w_{j} C_{j}$, Goemans, Wein and Williamson (cited as personal communication in [14]) noted that the preemptive version of the WSPT rule is 2-competitive; it schedules at any point in time the highest-priority job, possibly preempting jobs of lower priority. (A proof of this result is given in [14].) Our preemptive parallel-machine algorithm is the direct extension of this variation of Smith's rule. Schulz and Skutella [14] and Goemans et al. [5] give comprehensive reviews of the development of on-line algorithms for the preemptive and nonpreemptive single-machine problems, respectively; Hall et al. [7] do the same for the parallel machine counterparts. 
On the side of negative results, Vestjens [18] proved a universal lower bound of 1.309 for the competitive ratio of any deterministic on-line algorithm for $P\left|r_{j}\right| \sum C_{j}$. In the preemptive case, the currently known lower bound is $\frac{22}{21}$, also given by Vestjens.

Let us eventually mention that the currently best randomized on-line algorithms for the two problems considered here have (expected) competitive ratio 2; see [15]. Moreover, the off-line versions of these problems are well understood; in fact, both problems have a polynomial-time approximation scheme [1].

\section{Preemptive parallel machine scheduling}

We consider the following extension of the single-machine preemptive WSPT rule to identical parallel machines.

\section{Algorithm 1: P-WSPT}

At any point in time, schedule the $m$ jobs with the highest priorities among the available, not yet completed jobs (or fewer if less than $m$ incomplete jobs are available). Interrupt the processing of currently active jobs, if necessary.

The algorithm works on-line since the decision about which job to run at any given point in time $t$ is just based on the set of available jobs at time $t$. In fact, it only depends on the priorities of the available jobs. In particular, Algorithm P-WSPT also operates in an environment in which actual job weights and processing times may not become available before the completion of the jobs, as long as the jobs' priorities are known at their respective release dates.

Theorem 2.1. The Algorithm P-WSPT produces a solution of objective function value at most twice the optimal value for the off-line problem $P\left|r_{j}, p m t n\right| \sum w_{j} C_{j}$.

Proof. Consider the time interval $\left(r_{j}, C_{j}\right]$ for an arbitrary but fixed job $j$. We partition this interval into two disjunctive sets of subintervals, which we call $I(j)$ and $\bar{I}(j)$, respectively. We let $I(j)$ contain the subintervals in which job $j$ is being processed; $\bar{I}(j)$ denotes the set of remaining subintervals. Note that no machine can be idle during the subintervals belonging to $\bar{I}(j)$. Since the algorithm processes job $j$ after its release date $r_{j}$ whenever a machine is idle, we obtain

$C_{j} \leqslant r_{j}+|I(j)|+|\bar{I}(j)|$,

where $|\cdot|$ denotes the sum of the lengths of the subintervals in the corresponding set.

The overall length of $I(j)$ is clearly $p_{j}$. Only jobs with a higher ratio of weight to processing time than $j$ can be processed during the intervals of the set $\bar{I}(j)$, because the algorithm gives priority to $j$ before scheduling jobs with lower ratio. In the worst case, that is when $|\bar{I}(j)|$ is maximal, all jobs with higher priority than $j$ are being processed in the subintervals of this set. Then $|\bar{I}(j)|=\left(\sum_{k<j} p_{k}\right) / m$, and we can bound the value of the P-WSPT schedule as follows: $\sum_{j} w_{j} C_{j} \leqslant \sum_{j} w_{j}\left(r_{j}+p_{j}\right)+\sum_{j} w_{j} \sum_{k<j} \frac{p_{k}}{m}$.

Since the completion time $C_{j}$ of a job $j$ is always at least as large as its release date plus its processing time, $\sum_{j} w_{j}\left(r_{j}+p_{j}\right)$ is obviously a lower bound on the value of an optimal schedule. Moreover, $\sum_{j} w_{j} \sum_{k \leqslant j} p_{k} / m$ is the objective function value of an optimal solution to the corresponding instance of the relaxed problem $1 \| \mid \sum w_{j} C_{j}$ on a single machine with speed $m$ times the speed of any of the identical parallel machines. As this problem is indeed a relaxation of the scheduling problem considered here, we can conclude that the P-WSPT algorithm is 2-competitive.

A family of instances provided by Schulz and Skutella [14] shows that this result cannot be improved. In fact, for $m=1$, P-WSPT coincides with the preemptive single-machine algorithm studied in their paper. Taking $m$ copies of Schulz and Skutella's instance yields the following result.

Lemma 2.2. The competitive ratio of the Algorithm P-WSPT is not better than 2 for the on-line problem $P\left|r_{j}, p m t n\right| \sum w_{j} C_{j}$, for any given number of $m a-$ chines.

Proof. We include a proof for the sake of completeness. We consider an instance that is slightly different from the one given in [14]. It consists of $m$ copies of $n+1$ jobs with $w_{j}=1, p_{j}=n-j / n$ and $r_{j}=j n-$ 
$j(j+1) /(2 n)$ for all $0 \leqslant j \leqslant n$. Algorithm P-WSPT preempts any job when it has left just $1 / n$ units of processing time and finishes it only after all jobs with a larger release date have been completed. The value of this schedule is $m\left(\sum_{j=0}^{n}\left(r_{n}+p_{n}+j / n\right)\right)$. An optimal off-line algorithm does not preempt any job and yields a schedule of value $m\left(\sum_{j=0}^{n}\left(r_{j}+p_{j}+j / n\right)\right)$. A simple calculation shows that the ratio of the values of the P-WSPT schedule and the optimal schedule goes to 2 when $n$ goes to infinity.

Of course, Theorem 2.1 subsumes the scheduling problem $P\left|r_{j}, \mathrm{pmtn}\right| \sum C_{j}$ as a special case. Thus, this extension of the 2-competitive single-machine shortest processing time (SPT) rule to the parallel machine setting has the same competitive ratio as the analogous extension of Schrage's optimal single-machine SRPT rule [12].

\section{Nonpreemptive parallel machine scheduling}

Every reasonable on-line algorithm for nonpreemptive scheduling has to make use of some kind of waiting strategy. We refer the reader to [18, Chapter 2] and [11] for comprehensive discussions of related techniques for the single machine. Here, we extend the idea of delaying release dates to the parallel machine problem.

\section{Algorithm 2: SHIFTED WSPT}

Modify the release date of every job $j$ to $r_{j}^{\prime}$, where $r_{j}^{\prime}$ is some value between $\max \left\{r_{j}, \alpha p_{j}\right\}$ and $r_{j}+\alpha p_{j}$, for some $\alpha \in(0,1]$. Whenever a machine becomes idle, choose among the available jobs a job $j$ with highest priority and schedule it on the idle machine.

Note that this is indeed an on-line algorithm; we will later choose $\alpha$ to minimize the corresponding competitive ratio. Moreover, for $m=1$ and $\alpha=1$, Algorithm SHIFTED WSPT is identical to the algorithm proposed in [11] for $1\left|r_{j}\right| \sum C_{j}$. The idea of shifting release dates to ensure that the processing time of any one job is not too large compared to its arrival time is also present in the proposed approximation schemes for this class of problems [1].
In the analysis of the SHIFTED WSPT algorithm, we make use of the following lower bound on the optimal value of the relaxed problem with trivial release dates, which is due to Eastman et al. [4].

Lemma 3.1. The value of an optimal schedule for an instance of the scheduling problem $P \| \sum w_{j} C_{j}$ is bounded from below by

$\sum_{j=1}^{n} w_{j} \sum_{k \leqslant j} \frac{p_{k}}{m}+\frac{m-1}{2 m} \sum_{j=1}^{n} w_{j} p_{j}$.

Let us now analyze the performance of the SHIFTED WSPT algorithm.

Theorem 3.2. The Algorithm SHIFTED WSPT has a competitive ratio of less than $2+\max \{1 / \alpha, \alpha+(m-$ 1)/2m\} for the on-line problem $P\left|r_{j}\right| \sum w_{j} C_{j}$.

Proof. The algorithm schedules a job $j$ at time $r_{j}^{\prime}$ if a machine is idle and $j$ is the job with the highest ratio of weight to processing time among all available jobs; otherwise, $j$ has to wait for some time. The waiting time for $j$ after $r_{j}^{\prime}$ is caused by two types of jobs: jobs with lower priority that started before time $r_{j}^{\prime}$, and jobs with higher priority. Note that the algorithm does not insert idle time on any machine in the time interval between $r_{j}^{\prime}$ and the start of job $j$.

Clearly, every machine has at most one low-priority job $\ell>j$ that is running at time $r_{j}^{\prime}$ and before the start of job $j$. By construction, such a job $\ell$ satisfies $\alpha p_{\ell} \leqslant r_{\ell}^{\prime}<r_{j}^{\prime}$. Thus, it is completed by time $(1+$ $1 / \alpha) r_{j}^{\prime}$. Consequently, any job that is running between $(1+1 / \alpha) r_{j}^{\prime}$ and the start of job $j$ must have a higher priority than $j$. The total processing time of these jobs is bounded by $\sum_{h<j} p_{h}$. As a result, one of the $m$ parallel machines finishes processing jobs of this kind after at most $\sum_{h<j} p_{h} / m$ time units. Hence,

$$
\begin{aligned}
C_{j}< & \left(1+\frac{1}{\alpha}\right) r_{j}^{\prime}+\sum_{h<j} \frac{p_{h}}{m}+p_{j} \\
\leqslant & \left(1+\frac{1}{\alpha}\right)\left(r_{j}+\alpha p_{j}\right)+\frac{m-1}{2 m} p_{j} \\
& +\sum_{h \leqslant j} \frac{p_{h}}{m}+\frac{m-1}{2 m} p_{j}
\end{aligned}
$$




$$
\begin{aligned}
= & \left(\frac{r_{j}}{\alpha}+\left(\alpha+\frac{m-1}{2 m}\right) p_{j}\right) \\
& +\left(r_{j}+p_{j}\right)+\sum_{h \leqslant j} \frac{p_{h}}{m}+\frac{m-1}{2 m} p_{j} .
\end{aligned}
$$

Thus, Algorithm SHIFTED WSPT generates a schedule of value

$$
\begin{aligned}
& \sum_{j} w_{j} C_{j}<\sum_{j} w_{j}\left(r_{j}+p_{j}\right) \\
& \cdot\left(1+\max \left\{\frac{1}{\alpha}, \alpha+\frac{m-1}{2 m}\right\}\right) \\
&+\sum_{j} w_{j}\left(\sum_{h \leqslant j} \frac{p_{h}}{m}+\frac{m-1}{2 m} p_{j}\right) .
\end{aligned}
$$

The proof is completed by applying two lower bounds on the optimal value: first, the trivial lower bound $\sum_{j} w_{j}\left(r_{j}+p_{j}\right)$, and second, the lower bound presented in Lemma 3.1.

A simple calculation shows that the minimum of $\max \{1 / \alpha, \alpha+(m-1) / 2 m\}$ is attained at $\alpha=(1-m+$ $\left.\sqrt{16 m^{2}+(m-1)^{2}}\right) /(4 m)=: \alpha_{m}$. In particular, $\alpha_{1}=1$.

Corollary 3.3. The Algorithm SHIFTED WSPT with $\alpha=\alpha_{m}$ is $\left(2+1 / \alpha_{m}\right)$-competitive. The value of this increasing function of $m$ is 3 for the single-machine case and has its limit at $(9+\sqrt{17}) / 4 \approx 3.28$ for $m \rightarrow \infty$.

Lemma 3.4. Algorithm SHIFTED WSPT cannot achieve a better competitive ratio than $\max \{2+$ $\alpha, 1+1 / \alpha\} \geqslant 2+\frac{\sqrt{5}-1}{2}$ for $\alpha \in(0,1]$, on any number of machines.

Proof. We give two instances from which the lower bound follows. Consider $2 m$ jobs released at time 0 ; half of the jobs are of type I and have unit processing times and weights $\varepsilon$, whereas the other half of the jobs, type II, have processing time $1+\varepsilon$ and unit weight. The algorithm modifies the release dates and schedules jobs of type I at time $t=\alpha$ first, one on each machine, followed by jobs of type II. The value of the schedule is $m(\alpha+1) \varepsilon+m(\alpha+2+\varepsilon)$. In the optimal schedule, jobs of type II start processing first, at time $t=0$, such that the value of the schedule is $m(1+\varepsilon)+m(2+\varepsilon) \varepsilon$.
For $\varepsilon \rightarrow 0$, the ratio between the value of the SHIFTED WSPT schedule and the optimal schedule goes to $2+\alpha$.

The second instance consists again of $2 m$ jobs: half of the jobs are of type I and have release dates $1+\varepsilon$, processing times $\varepsilon$ and weights $1 / m$, whereas the other half of the jobs, type II, are released at time 0 and have processing time $1 / \alpha$ and zero weight. SHIFTED WSPT starts scheduling jobs at time 1 and obtains a solution with a value of at least $1+1 / \alpha+\varepsilon$. The value of the optimal schedule is $1+2 \varepsilon$.

For the special choice $\alpha=\alpha_{m}$, the first lower bound is tighter and it follows more concretely:

Corollary 3.5. The performance guarantee of Algorithm SHIFTED WSPT with $\alpha=\alpha_{m}$ is not better than $\left(1+7 m+\sqrt{16 m^{2}+(m-1)^{2}}\right) /(4 m)$ for instances with $m$ machines. This means that the above analysis for this specific algorithm has a gap of at most ( $m-$ $1) / 2 m<0.5$, and it is tight for the single-machine problem.

\section{References}

[1] F.N. Afrati, E. Bampis, C. Chekuri, D.R. Karger, C. Kenyon, S. Khanna, I. Milis, M. Queyranne, M. Skutella, C. Stein, M. Sviridenko, Approximation schemes for minimizing average weighted completion time with release dates, in: Proceedings of the 40th Annual IEEE Symposium on Foundations of Computer Science (FOCS), New York, 1999, pp. 32-43.

[2] E.J. Anderson, C.N. Potts, On-line scheduling of a single machine to minimize total weighted completion time, in: Proceedings of the Thirteenth Annual ACM-SIAM Symposium on Discrete Algorithms (SODA), San Francisco, CA, 2002, pp. 548-557.

[3] C. Chekuri, R. Motwani, B. Natarajan, C. Stein, Approximation techniques for average completion time scheduling, SIAM J. Comput. 31 (2001) 146-166.

[4] W.L. Eastman, S. Even, I.M. Isaacs, Bounds for the optimal scheduling of $n$ jobs on $m$ processors, Management Sci. 11 (1964) 268-279.

[5] M.X. Goemans, M. Queyranne, A.S. Schulz, M. Skutella, Y. Wang, Single machine scheduling with release dates, SIAM J. Discrete Math. 15 (2002) 165-192.

[6] R.L. Graham, E.L. Lawler, J.K. Lenstra, A.H.G. Rinnooy Kan, Optimization and approximation in deterministic sequencing and scheduling: a survey, Ann. Discrete Math. 5 (1979) 287-326.

[7] L.A. Hall, A.S. Schulz, D.B. Shmoys, J. Wein, Scheduling to minimize average completion time: off-line and on-line approximation algorithms, Math. Oper. Res. 22 (1997) 513-544. 
[8] J.A. Hoogeveen, A.P.A. Vestjens, Optimal on-line algorithms for single-machine scheduling, in: W.H. Cunningham, S.T. McCormick, M. Queyranne (Eds.), Proceedings of the Fifth Conference on Integer Programming and Combinatorial Optimization (IPCO), Lecture Notes in Computer Science, Vol. 1084, Springer, Berlin, 1996, pp. 404-414.

[9] J. Labetoulle, E.L. Lawler, J.K. Lenstra, A.H.G. Rinnooy Kan, Preemptive scheduling of uniform machines subject to release dates, in: W.R. Pulleyblank (Ed.), Progress in Combinatorial Optimization, Academic Press, New York, 1984, pp. 245-261.

[10] J.K. Lenstra, A.H.G. Rinnooy Kan, P. Brucker, Complexity of machine scheduling problems, Ann. Discrete Math. 1 (1977) 343-362.

[11] X. Lu, R.A. Sitters, L. Stougie, A class of on-line scheduling algorithms to minimize total completion time, Oper. Res. Lett. 31 (2003) 232-236.
[12] C.A. Phillips, C. Stein, J. Wein, Minimizing average completion time in the presence of release dates, Math. Program. 82 (1998) 199-223.

[13] L. Schrage, A proof of the optimality of the shortest remaining processing time discipline, Oper. Res. 16 (1968) 687-690.

[14] A.S. Schulz, M. Skutella, The power of $\alpha$-points in preemptive single machine scheduling, J. Sched. 5 (2002) 121-133.

[15] A.S. Schulz, M. Skutella, Scheduling unrelated machines by randomized rounding, SIAM J. Discrete Math. 15 (2002) 450-469.

[16] D.D. Sleator, R.E. Tarjan, Amortized efficiency of list update and paging rules, Comm. ACM 28 (1985) 202-208.

[17] W.E. Smith, Various optimizers for single-stage production, Naval Res. Logist. Quart. 3 (1956) 59-66.

[18] A.P.A. Vestjens, On-line machine scheduling, Ph.D. Thesis, Eindhoven University of Technology, Netherlands, 1997. 